\title{
Influence of Dyeing Conditions on Colour Strength and Colour Co- Ordinates of Silk Yarn Dyed with Acacia nilotica Pods
}

\author{
Dilshad Jamadar ${ }^{1}$, K.J. Sannapapamma ${ }^{2 *}$ and B. Kasturiba ${ }^{3}$ \\ ${ }^{1}$ University of Agricultural Sciences, Dharwad 580 005, Karnataka, India \\ ${ }^{2}$ AICRP HSc (CT), MARS, University of Agricultural Sciences, Dharwad-580005, \\ Karnataka, India \\ ${ }^{3}$ Department of Food Science and Nutrition, College of Community Science, University of \\ Agricultural Sciences, Dharwad-580005, Karnataka, India \\ *Corresponding author
}

\begin{tabular}{l} 
K e y w o r d s \\
$\begin{array}{l}\text { Acacia nilotica pods, } \\
\text { Colour co-ordinates, } \\
\text { Degummed mulberry silk, } \\
\text { K/S value, Reflectance } \\
\text { value, Mordants }\end{array}$ \\
Article Info \\
$\begin{array}{l}\text { Accepted: } \\
26 \text { July } 2018 \\
\text { Available Online: } \\
10 \text { August } 2018\end{array}$ \\
\hline
\end{tabular}

Natural dyes produce special aesthetic qualities which are environmentally friendly, add value to textile production as craftwork and as an industry. Today, many are rediscovering the joy of achieving colour through the use of renewable, non-toxic, natural sources. The dried Acacia nilotica pods were powdered by using traditional pounding technique and was soaked overnight in plain water with varied concentrations $(5,10,15,20)$ and M.L.R $(1: 20,1: 30,1: 40)$. The soaked solution was subjected to aqueous extraction method to optimise the dye concentration by using colour strength and reflectance value. The optimised dye extract (10g, 1:30, and 30min) was further used for dyeing silk yarn. The results showed that, irrespective of mordants and mordanting methods myrobolan treated silk yarn dyed with alum(15\%), stannous chloride $(0.5 \%)$, copper and ferrous sulphate $(3.00 \%)$ in pre mordanting method showed significantly increased colour strength with decreased reflectance value resulting into darker shades i.e., olive green to brown colour. Acacia nilotica pods can effectively be used as natural dye source on silk for producing varied eye cooling, soothing colour and colour combinations with better colour fastness.

\section{Introduction}

Silk beautiful of all the textile fibres with a unique property of fineness, strength, hand and feel with great affinity for dyeing. No other textile fibres possess such a fine natural lustre, softness and comfort wear properties. Silk, all over the world is considered as antiallergic, eco-friendly, and a symbol of beauty and thus famous as 'Queen of Textile fibre' and it has a greater affinity for natural and synthetic dyes and better fastness properties (www.Ibef.org).

The environmental friendly natural dyes are enjoying resurgence in popularity as a result of concern with the carcinogenic, mutagenic and sensitizing characteristics of synthetic dyes. The ban of certain number of synthetic dyes has stimulated the entry of the golden era of natural dyes. Natural dyes/colorants derived from the flora and fauna are believed to be 
safe because of non-toxic, non-carcinogenic and biodegradable nature, hence are ecofriendly and user friendly. Natural dyes are obtained from various parts of the plants viz., leaves, fruits, flowers, seeds, bark, rind, roots, husk, nuts and shoots which are used for textile colouration since time immemorial (Singh, 2000).

Acacia nilotica is truly multipurpose nitrogen fixing leguminous tree in India commonly called as babul and is a source of Indian gum arabic and the gum collected from the trunk and branches was formerly used in paints and medicines. This is frequently used in calico printing and dyeing as a thickening agent (Abhishek, 2015). Acacia nilotica possesses numerous phytochemicals such as the pods contain gallic acid and it's Me-este-n-digallic acid and condensed tannins. Bark contains tannin (12-20\%), terpenoids, saponins and glycosides, phlobetannin, gallic acid, protocatechuic acid, pyrocatechol (+) catechin, (-) epigallocatechin-5, 7-digallate. Root contains octaconsanol, betulin, B-amyrin and B-sitosterol. Pyrocatechol exhibits potential anxiolytic, antinociceptive and antimicrobial properties (Malviya, 2011). Hence, the Acacia nilotica pods have been selected for colouration of silk with an objective; to optimise the dyeing conditions of Acacia nilotica pods and to assess the colour strength and colour co-ordinates of dyed silk yarn.

\section{Materials and Methods}

Influence of dyeing conditions on colour strength and colour co-ordinates of silk yarn dyed with Acacia nilotica pods were assessed statistically by using One way Anova.

Acacia nilotica pods (Plate. 1) were collected from local forest of molakalmuru taluk of Chitradurga district, Karnataka. The collected fresh pods were shade dried and crushed into fine powder by traditional pounding technique (Plate. 3). Multivoltine yellow race degummed mulberry silk (Plate. 2) was procured from Demonstration Cum Training Center (DCTC), Central Silk Board. Rayapur, Dharwad

\section{Optimization of dyeing conditions}

The powdered Acacia nilotica with varied concentration $(5,10,15$ and $20 \mathrm{~g})$ was soaked overnight in different M.L.R (1:20, 1:30 and $1: 40)$ and the dye was extracted by aqueous method to optimize the dye concentration (Plate. 4). The cooled dye extract was filtered by filter paper and the $\mathrm{pH}$ was recorded (Plate 5). The extract was subjected to UV spectro photometer to assess the reflectance and colour co-ordinates of dye source. The dyeing variables viz., M.L.R (1:20, 1:30 and 1:40), dye concentration $(5,10,15$ and $20 \mathrm{~g})$ and time of extraction $(30,45,60 \mathrm{~min})$ were optimized based on the reflectance, colour co-ordinates and K/S value. The optimized dye extract (10g, 1:30 M.L.R, 30 min) was further used for dyeing silk yarn.

\section{Dyeing of silk yarn}

The degummed mulberry silk yarn pre-treated with myrobolan (20g owf for $1 \frac{1 / 2}{2}$ hour) and mordanted with Potash alum (5, 10 and 15\%), Copper sulphate and ferrous sulphate (1, 2 and $3 \%)$ and stannous chloride $(0.5,1$ and $1.5 \%)$ in pre, simultaneous and post mordanting methods dyed with optimised dye concentration (10\% owf) in varied M.L.R (1:20, 1:30, 1:40) and dyeing time (30, 45 and $60 \mathrm{~min}$ ) intervals. Based on the colour strength (K/S) and colour co-ordinates, 30 min dyeing time and 1:40 M.L.R was optimised for further dyeing (Plate 6).

\section{Mordant combinations}

Irrespective of mordants, mordant concentration and mordanting methods, dyed 
silk yarn were subjected to spectral assessment. Based on the colour strength and colour co-ordinates pre-mordanted dyed silk yarn with alum (15\%), stannous (0.5\%), copper and ferrous (3\%) were used for mordant combinations.

Potash alum + Stannous chloride

Potash alum + Copper sulphate

Potash alum + Ferrous sulphate

Stannous chloride + Copper sulphate

Stannous chloride + Ferrous sulphate

Copper sulphate + Ferrous sulphate

\section{Results and Discussion}

Influence of dyeing conditions on colour strength and colour co-ordinates of silk yarn dyed with Acacia nilotica pods

The Table 1 (Fig. 1) showed that, Irrespective of liquor ratio and dyeing time, silk yarn dyed with $(10 \%)$ concentration possessed significantly greater strength and darker shades than the control sample (2.80/84.14). this may be due to presence of phenolic contents present in the dye source which yields darker shades. Silk in the protein fibres have $-\mathrm{NH}_{2}$ and $-\mathrm{COOH}$ groups on either sides of polymer chain. $\mathrm{OH}^{+}$groups of pyrocatechol and pyragallol reacts with $-\mathrm{NH}^{2}$ groups of fibre to form ionic bond and yield high colour strength.

Among the extraction time and M.L.R the highest colour strength of silk yarn dyed with 10 per cent was obtained in 1:30 M.L.R at 60 minutes dyeing time with deeper shades. In general, optimum colour strength was noticed in 1:30 M.L.R with different time intervals than the 1:20 and 1:40 M.L.R.

This may be due to every fibre has its own dye saturation value, upto which shades can be produced and beyond that it cannot accommodate more dye.
Influence of dyeing conditions on colour strength and colour co-ordinates of silk yarn pre-treated with myrobolan

The Table 2 (Fig. 2) indicated that, the control sample possessed significantly lesser K/S values (8.67) than the myrobolan treated silk samples, due to presence of tannin content in the myrobolan which enhances the dye fixing on the fabric. As the time of dyeing intervals increased (30-60 $\mathrm{min}$ ) the colour strength of the dyed silk samples slowly decreased i.e. maximum colour strength (29.95) of silk sample was found in 1:40 material to liquor ratio at 30 min dyeing time with darker shades (63.05) followed by $45 \mathrm{~min}$ (28.43) and 60 min (27.57). The reason could be that, during dyeing dye molecules are gradually absorbed and diffused in the fibre mordant system and form co-ordinated complex with dye. After saturation value of dyeing, further dye absorption ceases and if heating is continued after that, the desorption starts and hence colour yield reduces.

Influence of dyeing conditions on colour strength and colour co-ordinates of silk yarn mordanted with potash alum

Colour strength is an indicator for accessing the absorption rate of dyed samples. it is inferred from the Table 3 (Fig. 3), among the different mordant concentrations and mordanting methods, potash alum premordanted dyed silk showed significantly greater colour strength in all the concentrations $(16.96 / 15 \%, 15.38 / 10 \%$ and $14.66 / 5 \%$ ) compared to simultaneous and post mordanting methods. This may be due to, application of heat during simultaneous and post mordanting supplies more energy usually facilitates higher rate of dye transportation and breaking of fibre-mordants-dye complexes leads to lower dye uptake and less colour strength. The results are in agreement with earlier studies of Konar (2014) who stated 
that, the colour strength of dyed silk samples reduced simultaneous and post mordanting method due to heat application. However, a* and $b^{*}$ values of pre-mordanted silk yarn showed more redder $(4.99 / 5 \%)$ and yellower $(33.48 / 5 \%)$ than the simultaneous and post mordanting method. This may be due to, the combined effect of pre-treatment i.e., silk yarn pre-treated with myrobolan and alum gave a synergistic effect and enhance the hue.

Influence of dyeing conditions on colour strength and colour co-ordinates of silk yarn mordanted with stannous chloride

It is observed from the Table 4 (Fig. 4) Colour strength and colour co-ordinates of silk yarn mordanted with stannous chloride possessed better dye yield, darker shades, more redder and yellower at lower concentrations in all the mordanting methods. This may be because of dyedsilk being exposed to air, oxidation takes place due to combined effects of silk polymer $\left(-\mathrm{NH}_{2}\right)$, dye phenols (Paracatechol \& Pyragallol) $\mathrm{OH}^{+}$and $\mathrm{SnCl}_{2}$. As the concentration of stannous chloride increased there was a decrease in colour strength, colour co-ordinates and tenacity. These results are on par with the results of Samanta and Konar (2010).

Influence of dyeing conditions on colour strength and colour co-ordinates silk yarn mordanted with copper sulphate

It is revealed from the Table 5 (Fig. 5) pretreated dyed silk mordanted with copper sulphate in all the concentrations exhibited higher colour strength due to a strong coordination between fibre-mordant-dye fibre leading to higher strength than the control sample. Whereas, lower strength was observed in simultaneous $(18.32 / 3 \%, 17.62 / 2 \%, 16.39 / 1$ $\%)$ and post-mordanting $(17.93 / 3 \%, 16.92 / 2 \%$, $16.25 / 1 \%$ ) methods. However, $\mathrm{a}^{*}$ and $\mathrm{b}^{*}$ values of pre-mordanted silk yarn showed more redder (5.78) and yellower (24.34) than the simultaneous and post mordanting methods. This can be attributed to fact that, the copper sulphate salt can form a ternary complex on the one side with the fibre of dyeing substitute and on the other side with the dye molecule. A strong co-ordination tendency can enhance the interaction between the fibre and the dye, resulting in high K/S values. This was supported by Khan et al., (2012).

Influence of dyeing conditions on colour strength and colour co-ordinates of silk yarn mordanted with ferrous sulphate

It is noticed from the Table 6 (Fig. 6) among the different mordant concentrations and mordanting methods, greater colour strength was noticed in ferrous sulphate pre-mordanted dyed silk in all the concentrations (141.54/3\%) compared to simultaneous $(88.53 / 3 \%)$ and post mordanting methods $(86.96 / 3 \%)$. This may be due to, iron salts such as ferrous sulphate as transition metal mordant form a large number of complexes with the dyemolecules, mostly octahedral ones with coordination number 6 . As a result, some coordination sites remain unoccupied when they interact with the fiber and at that time functional groups such as amino and carboxylic groups on the silk fiber can occupy these unoccupied sites. Thus, ferrous sulphate salts can form a ternary complex on one site with the fibre and in the other site with the dye. This resulted in higher dye uptake as well as shade change due to mordanting with ferrous sulphate (Uddin, 2014).

Irrespective of mordanting methods and mordant concentrations ferrous mordant concentration increased with decreasing colour co-ordinate values $\left(\mathrm{L}^{*}, \mathrm{a}^{*}, \mathrm{~b}^{*}\right)$ indicating darker shades $(27.56 / 3 \%)$ with more redder $(7.46 / 1 \%)$ and yellower $(10.48 / 1$ $\%)$. 


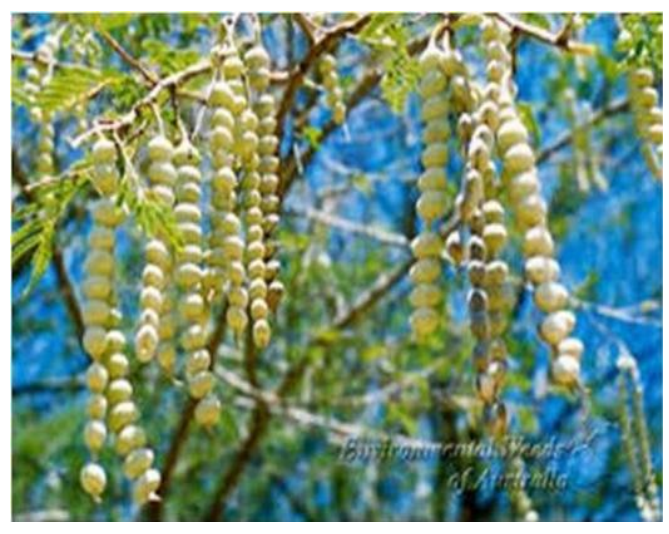

Plate 1. Acacia Nilotica Pods

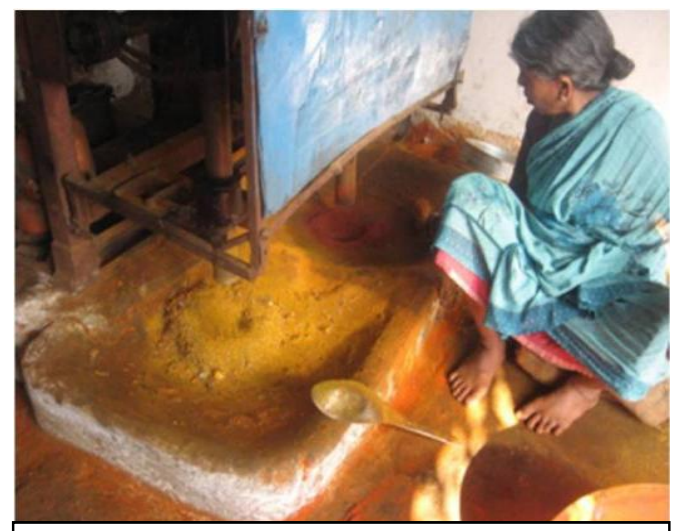

Plate 3. Pounding technique of Acacia Nilotica Pods

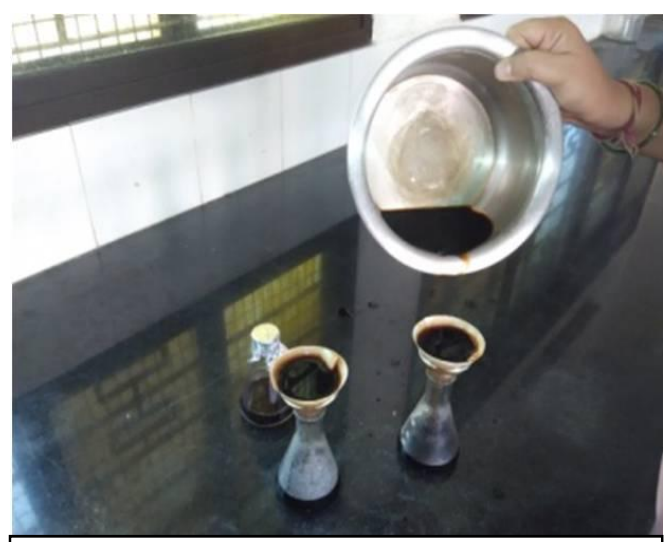

Plate 5. Acacia Nilotica dye filteration

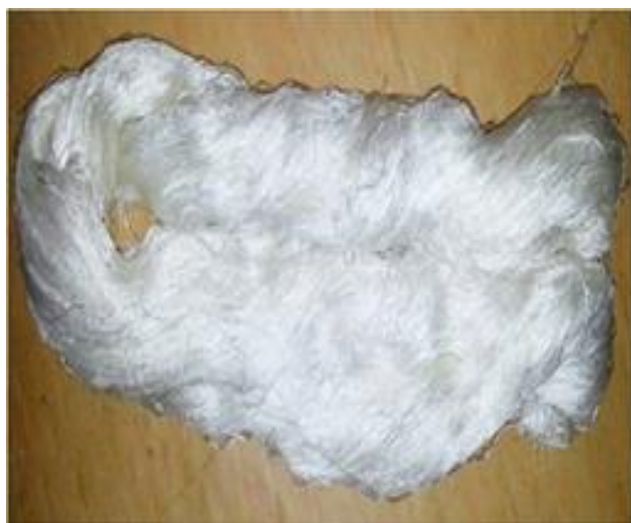

Plate 2. Degummed mulberry silk
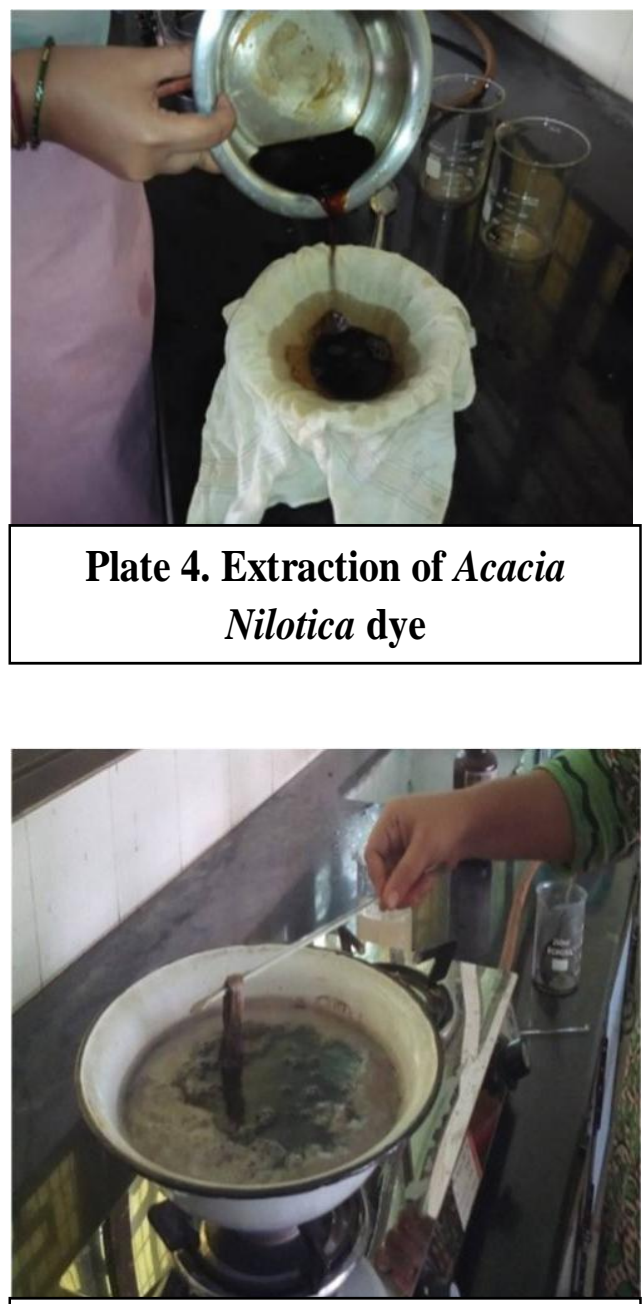

Plate 6. Dyeing silk yarn with Acacia Nilotica Pods 
Table.1 Influence of dyeing conditions on colour strength and colour co-ordinates of silk yarn dyed with Acacia nilotica pods

\begin{tabular}{|c|c|c|c|c|c|c|c|c|c|c|}
\hline \multirow{2}{*}{$\begin{array}{l}\text { Colour co- } \\
\text { ordinates }\end{array}$} & \multirow[t]{2}{*}{ Control } & \multicolumn{3}{|c|}{$1: 20$} & \multicolumn{3}{|c|}{$1: 30$} & \multicolumn{3}{|c|}{$1: 40$} \\
\hline & & $30 \mathrm{~min}$ & $45 \mathrm{~min}$ & $60 \mathrm{~min}$ & $30 \mathrm{~min}$ & $45 \mathrm{~min}$ & $60 \mathrm{~min}$ & $30 \mathrm{~min}$ & $45 \mathrm{~min}$ & $60 \mathrm{~min}$ \\
\hline $\mathbf{K} / \mathbf{S}$ & 2.80 & $\begin{array}{l}15.41 \\
(4.14)\end{array}$ & $\begin{array}{c}15.53 \\
(10.77)\end{array}$ & $\begin{array}{l}16.04 \\
(9.58)\end{array}$ & $\begin{array}{l}19.49 \\
(5.35)\end{array}$ & $\begin{array}{l}19.56 \\
(4.39)\end{array}$ & $\begin{array}{l}20.73 \\
(6.65)\end{array}$ & $\begin{array}{l}17.04 \\
(4.09)\end{array}$ & $\begin{array}{l}17.61 \\
(3.64)\end{array}$ & $\begin{array}{l}18.39 \\
(4.94)\end{array}$ \\
\hline $\mathbf{L}^{*}$ & 84.14 & $\begin{array}{l}67.47 \\
(4.17)\end{array}$ & $\begin{array}{l}71.79 \\
(3.47)\end{array}$ & $\begin{array}{l}74.61 \\
(4.16)\end{array}$ & $\begin{array}{l}71.35 \\
(3.06)\end{array}$ & $\begin{array}{l}72.94 \\
(4.15)\end{array}$ & $\begin{array}{l}75.46 \\
(7.46)\end{array}$ & $\begin{array}{l}68.77 \\
(3.47)\end{array}$ & $\begin{array}{l}70.68 \\
(2.18)\end{array}$ & $\begin{array}{l}71.32 \\
(4.29)\end{array}$ \\
\hline$a^{*}$ & 0.72 & $\begin{array}{c}5.66 \\
(-0.61)\end{array}$ & $\begin{array}{c}3.36 \\
(-2.31)\end{array}$ & $\begin{array}{c}4.72 \\
(-2.13)\end{array}$ & $\begin{array}{c}5.09 \\
(-0.63)\end{array}$ & $\begin{array}{c}5.87 \\
(0.19)\end{array}$ & $\begin{array}{c}4.69 \\
(-0.95)\end{array}$ & $\begin{array}{c}4.94 \\
(-0.73)\end{array}$ & $\begin{array}{c}5.49 \\
(-0.18)\end{array}$ & $\begin{array}{c}5.66 \\
(-0.78)\end{array}$ \\
\hline $\mathbf{b}^{*}$ & 11.21 & $\begin{array}{c}20.56 \\
(-0.51)\end{array}$ & $\begin{array}{c}13.21 \\
(-7.41)\end{array}$ & $\begin{array}{c}15.68 \\
(-5.11)\end{array}$ & $\begin{array}{c}20.01 \\
(-0.64)\end{array}$ & $\begin{array}{c}16.02 \\
(-4.07)\end{array}$ & $\begin{array}{c}13.97 \\
(-6.75)\end{array}$ & $\begin{array}{c}18.26 \\
(-2.62)\end{array}$ & $\begin{array}{c}17.85 \\
(-2.90)\end{array}$ & $\begin{array}{l}18.96 \\
(-0.34)\end{array}$ \\
\hline
\end{tabular}

Figures in parenthesis indicate $K / S(\Delta E), L *(\Delta L), a^{*}(\Delta a), b^{*}(\Delta b)$

K/S- Colour strength

L-The lightness/darkness co-ordinate

$a^{*}$ - The red/green co-ordinate with $+a^{*}$ indicating red $-a^{*}$ indicating green

$b^{*}$ - The yellow/blue co-ordinate with $+b^{*}$ indicating yellow and $-b^{*}$ indicating blue

ANOVA Table

\begin{tabular}{|c|c|c|c|c|c|c|c|c|c|}
\hline \multirow[b]{2}{*}{$\begin{array}{c}\text { Colour co- } \\
\text { ordinates }\end{array}$} & \multicolumn{3}{|c|}{$1: 20$} & \multicolumn{3}{|c|}{$1: 30$} & \multicolumn{3}{|c|}{$1: 40$} \\
\hline & S.Em. \pm & CD (5\%) & CV \% & S.Em. \pm & CD (5\%) & $\mathrm{CV} \%$ & S.Em. \pm & CD (5\%) & CV \% \\
\hline $\mathrm{K} / \mathrm{S}$ & 0.10 & $0.14^{*}$ & 1.69 & 0.09 & $0.13^{*}$ & 1.47 & 0.11 & $0.14 *$ & 1.73 \\
\hline $\mathbf{L *}$ & 0.38 & $0.49^{*}$ & 1.12 & 0.38 & $0.51^{*}$ & 1.12 & 0.30 & $0.41^{*}$ & 0.94 \\
\hline$a^{*}$ & 0.01 & $0.01 *$ & 0.70 & 0.01 & $0.01 *$ & 0.50 & 0.01 & $0.01 *$ & 0.33 \\
\hline$b^{*}$ & 0.22 & $0.30 *$ & 3.31 & 0.22 & $0.29 *$ & 3.24 & 0.27 & $0.36^{*}$ & 3.68 \\
\hline
\end{tabular}

*Significant@5\% level

Table.2 Influence of dyeing conditions on colour strength and colour co-ordinates of silk yarn pre-treated with myrobolan

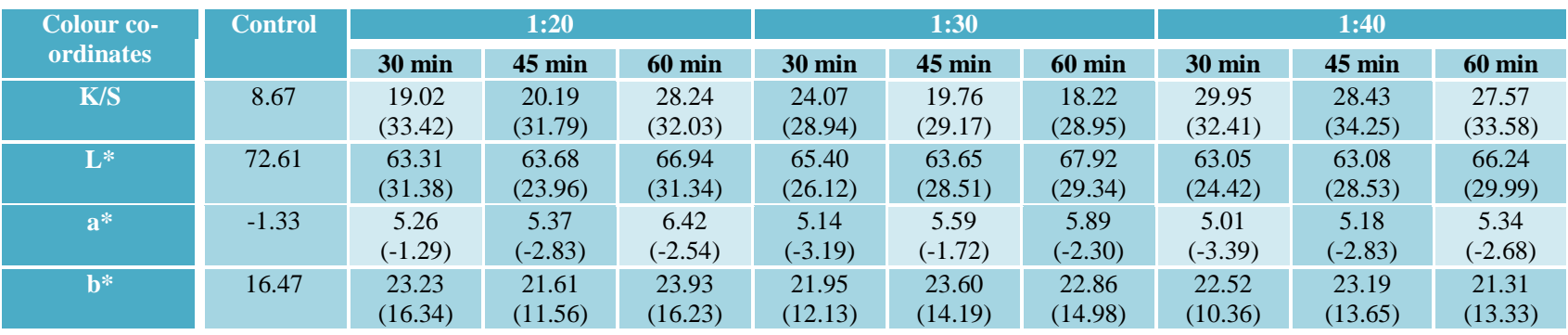

Figures in parenthesis indicate K/S $(\Delta \mathrm{E}), \mathrm{L}^{*}(\Delta \mathrm{L}), \mathrm{a}^{*}(\Delta \mathrm{a}), \mathrm{b}^{*}(\Delta \mathrm{b})$

K/S- Colour strength

L-The lightness/darkness co-ordinate

$a^{*}$ - The red/green co-ordinate with $+a^{*}$ indicating red $-a^{*}$ indicating green

$\mathrm{b}^{*}$ - The yellow/blue co-ordinate with $+\mathrm{b}^{*}$ indicating yellow and $-\mathrm{b}^{*}$ indicating blue

ANOVA Table

\begin{tabular}{|c|c|c|c|c|c|c|c|c|c|}
\hline & \multicolumn{3}{|c|}{$1: 20$} & \multicolumn{3}{|c|}{ 1:30 } & \multicolumn{3}{|c|}{$1: 40$} \\
\hline $\begin{array}{l}\text { Colour co- } \\
\text { ordinates }\end{array}$ & S.Em. \pm & CD $(5 \%)$ & CV \% & S.Em. \pm & CD $(5 \%)$ & CV \% & S.Em. \pm & CD (5\%) & CV \% \\
\hline $\mathrm{K} / \mathrm{S}$ & 0.37 & $0.49 *$ & 5.29 & 0.37 & $0.49 *$ & 5.38 & 0.40 & $0.54^{*}$ & 6.12 \\
\hline $\mathbf{L}^{*}$ & 2.43 & $0.97 *$ & 0.72 & 2.23 & $0.90 *$ & 0.67 & 2.19 & $0.90 *$ & 0.65 \\
\hline$a^{*}$ & 0.32 & $0.44 *$ & 18.52 & 0.21 & $0.28^{*}$ & 12.25 & 0.22 & $0.29 *$ & 13.97 \\
\hline b* & 0.45 & $0.59 *$ & 4.69 & 0.42 & $0.56^{*}$ & 4.41 & 0.45 & $0.61^{*}$ & 4.87 \\
\hline
\end{tabular}

*Significant @ $5 \%$ level 
Table.3 Influence of dyeing conditions on colour strength and colour co-ordinates of silk yarn mordanted with potash alum

Dye concentration -10 per cent (owf) Dyeing time- 30 min $\quad$ M.L.R ratio -1:40

\begin{tabular}{|c|c|c|c|c|c|c|c|c|c|c|}
\hline \multirow[t]{2}{*}{$\begin{array}{l}\text { Colour co- } \\
\text { ordinates }\end{array}$} & \multirow[t]{2}{*}{$\begin{array}{c}\text { Contro } \\
\text { I }\end{array}$} & \multicolumn{3}{|c|}{ Pre-mordanting method } & \multicolumn{3}{|c|}{$\begin{array}{l}\text { Simultaneous mordanting } \\
\text { method }\end{array}$} & \multicolumn{3}{|c|}{ Post-mordanting method } \\
\hline & & $5 \%$ & $10 \%$ & $15 \%$ & $5 \%$ & $10 \%$ & $15 \%$ & $5 \%$ & $10 \%$ & $15 \%$ \\
\hline $\mathbf{K} / \mathbf{S}$ & 13.00 & $\begin{array}{c}14.66 \\
(37.77)\end{array}$ & $\begin{array}{c}15.38 \\
(37.26)\end{array}$ & $\begin{array}{c}16.96 \\
(38.17)\end{array}$ & $\begin{array}{c}14.09 \\
(35.53)\end{array}$ & $\begin{array}{c}14.15 \\
(32.24)\end{array}$ & $\begin{array}{c}15.11 \\
(33.28)\end{array}$ & $\begin{array}{c}14.04 \\
(32.58)\end{array}$ & $\begin{array}{c}14.16 \\
(35.09)\end{array}$ & $\begin{array}{c}14.50 \\
(35.01)\end{array}$ \\
\hline $\mathbf{L}^{*}$ & 71.86 & $\begin{array}{l}69.65 \\
(30.14)\end{array}$ & $\begin{array}{c}69.79 \\
(30.74)\end{array}$ & $\begin{array}{l}69.98 \\
(29.07)\end{array}$ & $\begin{array}{c}67.25 \\
(27.89)\end{array}$ & $\begin{array}{c}67.81 \\
(28.67)\end{array}$ & $\begin{array}{c}66.82 \\
(31.59)\end{array}$ & $\begin{array}{l}70.63 \\
(31.49)\end{array}$ & $\begin{array}{c}70.94 \\
(30.97)\end{array}$ & $\begin{array}{c}69.85 \\
(33.49)\end{array}$ \\
\hline a* & 6.18 & $\begin{array}{c}4.99 \\
(-3.43)\end{array}$ & $\begin{array}{c}3.78 \\
(-3.62)\end{array}$ & $\begin{array}{c}2.69 \\
(-5.05)\end{array}$ & $\begin{array}{c}3.16 \\
(-4.36)\end{array}$ & $\begin{array}{c}3.17 \\
(-4.28)\end{array}$ & $\begin{array}{c}2.18 \\
(-5.65)\end{array}$ & $\begin{array}{c}3.57 \\
(-3.74)\end{array}$ & $\begin{array}{c}2.42 \\
(-5.84)\end{array}$ & $\begin{array}{c}3.19 \\
(-5.32)\end{array}$ \\
\hline b* & 22.54 & $\begin{array}{c}33.48 \\
(-22.43)\end{array}$ & $\begin{array}{c}29.62 \\
(-20.29)\end{array}$ & $\begin{array}{l}27.97 \\
(17.40)\end{array}$ & $\begin{array}{c}24.78 \\
(15.32)\end{array}$ & $\begin{array}{c}25.20 \\
(15.23)\end{array}$ & $\begin{array}{l}25.16 \\
(15.81)\end{array}$ & $\begin{array}{c}23.84 \\
(14.11)\end{array}$ & $\begin{array}{c}24.71 \\
(14.29)\end{array}$ & $\begin{array}{c}25.74 \\
(-16.14)\end{array}$ \\
\hline
\end{tabular}

Figures in parenthesis indicate K/S $(\Delta \mathrm{E}), \mathrm{L}^{*}(\Delta \mathrm{L}), \mathrm{a}^{*}(\Delta \mathrm{a}), \mathrm{b}^{*}(\Delta \mathrm{b}) ; \mathrm{K} / \mathrm{S}$ - Colour strength

L-The lightness/darkness co-ordinate

$a^{*}$ - The red/green co-ordinate with $+a^{*}$ indicating red $-a^{*}$ indicating green

$\mathrm{b}^{*}$ - The yellow/blue co-ordinate with $+\mathrm{b}^{*}$ indicating yellow and $-\mathrm{b}^{*}$ indicating blue

\section{ANOVA Table}

\begin{tabular}{|c|c|c|c|c|c|c|c|c|c|}
\hline & \multicolumn{3}{|c|}{ Pre-mordanting method } & \multicolumn{3}{|c|}{ Simultaneous mordanting method } & \multicolumn{3}{|c|}{ Post-mordanting method } \\
\hline $\begin{array}{c}\text { Colour co- } \\
\text { ordinates }\end{array}$ & S.Em. \pm & CD (5 \%) & CV \% & S.Em. \pm & CD (5 \%) & CV \% & S.Em. \pm & CD (5 \%) & CV \% \\
\hline K/S & 0.24 & $0.32^{*}$ & 3.53 & 0.22 & $0.30^{\mathrm{NS}}$ & 3.53 & 0.19 & $0.26^{\mathrm{NS}}$ & 3.08 \\
\hline L $^{*}$ & 0.54 & $0.72^{\mathrm{NS}}$ & 1.74 & 0.64 & $0.86^{\mathrm{NS}}$ & 2.10 & 0.59 & $0.80^{\mathrm{NS}}$ & 1.93 \\
\hline $\mathbf{a}^{*}$ & 0.34 & $0.46^{\mathrm{NS}}$ & 17.33 & 0.30 & $0.40^{\mathrm{NS}}$ & 18.37 & 0.25 & $0.34^{\mathrm{NS}}$ & 14.70 \\
\hline $\mathbf{b}^{*}$ & 0.46 & $0.62^{\mathrm{NS}}$ & 3.62 & 0.38 & $0.52^{\mathrm{NS}}$ & 3.53 & 0.40 & $0.54^{\mathrm{NS}}$ & 3.73 \\
\hline
\end{tabular}

NS- Non Significant; *Significant @ $5 \%$ level

Table.4 Influence of dyeing conditions on colour strength and colour co-ordinates of silk yarn mordanted with stannous chloride

Dye concentration -10 per cent (owf)

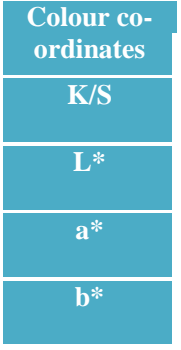

\begin{tabular}{|c|}
\hline Control \\
\hline 21.93 \\
\hline 74.89 \\
\hline 6.00 \\
\hline 21.93 \\
\hline
\end{tabular}

Pre-mordanting method

$0.5 \%$

\begin{tabular}{|l|l|r|}
\hline 29.72 & 29.27 & 27.79 \\
\hline
\end{tabular}

\begin{tabular}{|l|c|c|}
$(32.15)$ & $(36.45)$ & $(36.79)$ \\
\hline
\end{tabular}

\begin{tabular}{|l|l|l|}
\hline 63.12 & 68.72 & 71.35 \\
\hline
\end{tabular}

\begin{tabular}{|c|c|c|}
$(24.57)$ & $(30.55)$ & $(31.88)$ \\
\hline 5.75 & 4.20 & 3.92
\end{tabular}

\begin{tabular}{l|l|r}
\hline 5.75 & 4.20 & 3.92
\end{tabular}

\begin{tabular}{l|l|r|}
$(-1.58)$ & $(-3.47)$ & $(-3.48)$ \\
\hline
\end{tabular}

\begin{tabular}{|l|l|l|}
29.72 & 29.28 & 27.79 \\
\hline
\end{tabular}
Dyeing time- 30 min

\begin{tabular}{|c|c|c|c|c|c|}
\hline $0.5 \%$ & $1.0 \%$ & $1.5 \%$ & $0.5 \%$ & $1.0 \%$ & $1.5 \%$ \\
\hline 25.33 & 25.04 & 24.90 & 26.01 & 24.31 & 22.10 \\
$(33.53)$ & $(39.10)$ & $(37.45)$ & $(35.33)$ & $(33.43)$ & $(35.81)$ \\
\hline 67.40 & 68.05 & 73.13 & 67.42 & 71.23 & 71.74 \\
$(-29.36)$ & $(29.13)$ & $(-35.77)$ & $(-32.68)$ & $(-32.80)$ & $(-33.77)$ \\
\hline 4.69 & 2.88 & 2.80 & 4.20 & 3.98 & 3.08 \\
$(-3.03)$ & $(-5.16)$ & $(-4.52)$ & $(-3.02)$ & $(-3.15)$ & $(-4.11)$ \\
\hline 25.33 & 25.04 & 24.90 & 26.01 & 24.31 & 22.10 \\
$(-15.27)$ & $(-14.89)$ & $(-15.45)$ & $(-12.09)$ & $(-15.94)$ & $(13.96)$ \\
\hline
\end{tabular}

Figures in parenthesis indicate K/S $(\Delta \mathrm{E}), \mathrm{L}^{*}(\Delta \mathrm{L}), \mathrm{a}^{*}(\Delta \mathrm{a}), \mathrm{b}^{*}(\Delta \mathrm{b}) ; \mathrm{K} / \mathrm{S}$ - Colour strength

L-The lightness/darkness co-ordinate

$a^{*}$ - The red/green co-ordinate with $+a^{*}$ indicating red $-a^{*}$ indicating green

$b^{*}$ - The yellow/blue co-ordinate with $+b^{*}$ indicating yellow and $-b^{*}$ indicating blue

\section{ANOVA Table}

\begin{tabular}{|c|c|c|c|c|c|c|c|c|c|}
\hline & \multicolumn{3}{|c|}{ Pre-mordanting method } & \multicolumn{3}{|c|}{ Simultaneous mordanting method } & \multicolumn{3}{|c|}{ Post-mordanting method } \\
\hline $\begin{array}{c}\text { Colour co- } \\
\text { ordinates }\end{array}$ & S.Em. \pm & CD $(5 \%)$ & CV \% & S.Em. \pm & CD $(5 \%)$ & $\mathrm{CV} \%$ & S.Em. \pm & CD $(5 \%)$ & CV \% \\
\hline $\mathrm{K} / \mathrm{S}$ & 0.29 & $0.40 *$ & 2.45 & 0.29 & $0.39 *$ & 2.72 & 0.26 & $0.35^{*}$ & 2.46 \\
\hline $\mathbf{L}^{*}$ & 0.30 & $0.41 *$ & 1.00 & 0.22 & $0.29 *$ & 0.69 & 0.29 & $0.39 *$ & 0.95 \\
\hline$a^{*}$ & 0.22 & $0.29 *$ & 9.84 & 0.18 & $0.24 *$ & 10.07 & 0.19 & $0.25^{*}$ & 9.79 \\
\hline $\mathbf{b}^{*}$ & 0.29 & $0.40^{*}$ & 2.45 & 0.29 & $0.39 *$ & 2.72 & 0.26 & $0.35^{*}$ & 2.46 \\
\hline
\end{tabular}

*Significant @ $5 \%$ level 
Table.5 Influence of dyeing conditions on colour strength and colour co-ordinates silk yarn mordanted with copper sulphate

Dye concentration -10 per cent (owf)

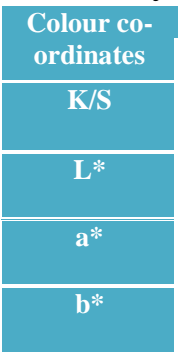

Dyeing time- 30 min

Simultaneous mordanting method

\begin{tabular}{|c|c|c|}
\hline $1 \%$ & $2 \%$ & $3 \%$ \\
\hline 16.39 & 17.62 & 18.32 \\
$(30.36)$ & $(28.33)$ & $(26.17)$ \\
\hline 66.39 & 64.81 & 61.36 \\
$(-27.77)$ & $(-25.72)$ & $(-22.68)$ \\
\hline 4.44 & 5.45 & 4.37 \\
$(-2.62)$ & $(-3.04)$ & $(-2.91)$ \\
\hline 21.99 & 21.77 & 21.79 \\
$(-11.94)$ & $(-11.44)$ & $(-12.51)$ \\
\hline
\end{tabular}

$(-13.95) \quad(-11.94)$

$(-11.44)$

$(-12.51)$
M.L.R ratio $-1: 40$

\begin{tabular}{|c|c|c|}
\hline \multicolumn{3}{|c|}{ Post-mordanting method } \\
\hline $1 \%$ & $2 \%$ & $3 \%$ \\
\hline 16.25 & 16.92 & 17.93 \\
$(29.79)$ & $(28.91)$ & $(29.46)$ \\
\hline 66.28 & 65.88 & 65.76 \\
$(-27.23)$ & $(-26.69)$ & $(26.85)$ \\
\hline 4.97 & 4.92 & 4.59 \\
$(-2.56)$ & $(-2.99)$ & $(-2.87)$ \\
\hline 22.24 & 21.39 & 22.32 \\
$(-11.78)$ & $(-10.52)$ & $(-11.69)$ \\
\hline
\end{tabular}

Figures in parenthesis indicate $\mathrm{K} / \mathrm{S}(\Delta \mathrm{E}), \mathrm{L} *(\Delta \mathrm{L}), \mathrm{a}^{*}(\Delta \mathrm{a}), \mathrm{b} *(\Delta \mathrm{b})$; K/S- Colour strength

L-The lightness/darkness co-ordinate

$\mathrm{a}^{*}$ - The red/green co-ordinate with $+\mathrm{a}^{*}$ indicating red $-\mathrm{a}^{*}$ indicating green

$\mathrm{b}^{*}$ - The yellow/blue co-ordinate with $+\mathrm{b}^{*}$ indicating yellow and $-\mathrm{b}^{*}$ indicating blue

ANOVA Table

\begin{tabular}{|c|c|c|c|c|c|c|c|c|c|}
\hline & \multicolumn{3}{|c|}{ Pre-mordanting method } & \multicolumn{3}{|c|}{ Simultaneous mordanting method } & \multicolumn{3}{|c|}{ Post-mordanting method } \\
\hline $\begin{array}{c}\text { Colour co- } \\
\text { ordinates }\end{array}$ & S.Em. \pm & CD (5\%) & CV \% & S.Em. \pm & CD (5\%) & CV \% & S.Em. \pm & CD (5\%) & CV \% \\
\hline $\mathrm{K} / \mathrm{S}$ & 0.26 & $0.34 *$ & 2.56 & 0.12 & $0.17 *$ & 1.66 & 0.23 & $0.31 *$ & 3.14 \\
\hline $\mathrm{L}^{*}$ & 0.18 & $0.24 *$ & 0.64 & 0.18 & $0.24 *$ & 0.61 & 0.27 & $0.36^{*}$ & 0.89 \\
\hline$a^{*}$ & 0.23 & $0.31^{\mathrm{NS}}$ & 9.41 & 0.26 & $0.35^{*}$ & 12.66 & 0.23 & $0.31^{\mathrm{NS}}$ & 10.33 \\
\hline $\mathbf{b}^{*}$ & 0.22 & $0.29 *$ & 2.11 & 0.21 & $0.29^{\mathrm{NS}}$ & 2.17 & 0.29 & $0.39^{\mathrm{NS}}$ & 2.97 \\
\hline
\end{tabular}

NS- Non Significant; *Significant @ $5 \%$ level

Table.6 Influence of dyeing conditions on colour strength and colour co-ordinates of silk yarn mordanted with ferrous sulphate

\begin{tabular}{|c|c|c|c|c|c|c|c|c|c|c|}
\hline \multicolumn{5}{|c|}{ Dye concentration -10 per cent (owf) } & \multicolumn{3}{|c|}{ Dyeing time- $30 \mathrm{~min}$} & \multicolumn{3}{|c|}{ M.L.R ratio $-1: 40$} \\
\hline \multirow[t]{2}{*}{$\begin{array}{l}\text { Colour co- } \\
\text { ordinates }\end{array}$} & \multirow[t]{2}{*}{ Control } & \multicolumn{3}{|c|}{ Pre-mordanting method } & \multicolumn{3}{|c|}{$\begin{array}{l}\text { Simultaneous mordanting } \\
\text { method }\end{array}$} & \multicolumn{3}{|c|}{ Post-mordanting method } \\
\hline & & $1 \%$ & $2 \%$ & $3 \%$ & $1 \%$ & $2 \%$ & $3 \%$ & $1 \%$ & $2 \%$ & $3 \%$ \\
\hline $\mathbf{K} / \mathbf{S}$ & 15.03 & $\begin{array}{l}77.58 \\
(2.34)\end{array}$ & $\begin{array}{l}97.41 \\
(7.60)\end{array}$ & $\begin{array}{l}141.54 \\
(13.18)\end{array}$ & $\begin{array}{l}69.28 \\
(3.824)\end{array}$ & $\begin{array}{l}88.09 \\
(6.90)\end{array}$ & $\begin{array}{c}88.53 \\
(10.19)\end{array}$ & $\begin{array}{l}55.01 \\
(11.49)\end{array}$ & $\begin{array}{l}64.80 \\
(6.64)\end{array}$ & $\begin{array}{l}86.96 \\
(8.38)\end{array}$ \\
\hline $\mathbf{L}^{*}$ & 67.08 & $\begin{array}{l}39.38 \\
(-0.91)\end{array}$ & $\begin{array}{l}33.06 \\
(-6.45)\end{array}$ & $\begin{array}{c}27.56 \\
(-11.31)\end{array}$ & $\begin{array}{l}41.44 \\
(-1.85)\end{array}$ & $\begin{array}{l}40.74 \\
(-1.93)\end{array}$ & $\begin{array}{c}33.10 \\
(-5.28)\end{array}$ & $\begin{array}{c}49.33 \\
(-10.12)\end{array}$ & $\begin{array}{c}39.34 \\
(-0.08)\end{array}$ & $\begin{array}{c}39.05 \\
(-2.88)\end{array}$ \\
\hline $\mathbf{a}^{*}$ & 2.32 & $\begin{array}{c}7.46 \\
(-0.13)\end{array}$ & $\begin{array}{c}6.88 \\
(-1.28)\end{array}$ & $\begin{array}{c}5.39 \\
(-1.85)\end{array}$ & $\begin{array}{c}5.18 \\
(-2.44)\end{array}$ & $\begin{array}{c}4.24 \\
(-3.31)\end{array}$ & $\begin{array}{c}3.61 \\
(-3.90)\end{array}$ & $\begin{array}{c}4.44 \\
(-3.04)\end{array}$ & $\begin{array}{c}3.75 \\
(-3.89)\end{array}$ & $\begin{array}{c}2.58 \\
(-4.72)\end{array}$ \\
\hline b* & 22.71 & $\begin{array}{l}10.48 \\
(-0.58)\end{array}$ & $\begin{array}{c}6.45 \\
(-3.65)\end{array}$ & $\begin{array}{c}3.94 \\
(-6.38)\end{array}$ & $\begin{array}{c}8.32 \\
(-1.89)\end{array}$ & $\begin{array}{c}5.18 \\
(-4.56)\end{array}$ & $\begin{array}{c}2.44 \\
(-7.68)\end{array}$ & $\begin{array}{c}12.45 \\
(-2.57)\end{array}$ & $\begin{array}{c}5.41 \\
(-4.91)\end{array}$ & $\begin{array}{c}4.34 \\
(-5.88)\end{array}$ \\
\hline
\end{tabular}

Figures in parenthesis indicate $\mathrm{K} / \mathrm{S}(\Delta \mathrm{E}), \mathrm{L} *(\Delta \mathrm{L}), \mathrm{a}^{*}(\Delta \mathrm{a}), \mathrm{b}^{*}(\Delta \mathrm{b}) ; \mathrm{K} / \mathrm{S}$ - Colour strength

L-The lightness/darkness co-ordinate

$a^{*}$ - The red/green co-ordinate with $+a^{*}$ indicating red $-a^{*}$ indicating green

$\mathrm{b}^{*}$ - The yellow/blue co-ordinate with $+\mathrm{b}^{*}$ indicating yellow and $-\mathrm{b}^{*}$ indicating blue

ANOVA Table

\begin{tabular}{|c|c|c|c|c|c|c|c|c|c|}
\hline & \multicolumn{3}{|c|}{ Pre-mordanting method } & \multicolumn{3}{|c|}{ Simultaneous mordanting method } & \multicolumn{3}{|c|}{ Post-mordanting method } \\
\hline $\begin{array}{c}\text { Colour co- } \\
\text { ordinates }\end{array}$ & S.Em. \pm & CD (5 \%) & CV \% & S.Em. \pm & CD (5 \%) & CV \% & S.Em. \pm & CD (5 \%) & CV \% \\
\hline K/S & 0.16 & $0.22^{*}$ & 0.44 & 0.11 & $0.14^{*}$ & 0.37 & 0.20 & $0.27^{*}$ & 0.81 \\
\hline L* & 0.19 & $0.27^{*}$ & 1.07 & 0.17 & $0.23^{*}$ & 0.85 & 0.15 & $0.21^{*}$ & 0.71 \\
\hline $\mathbf{a}^{*}$ & 0.19 & $0.26^{*}$ & 6.91 & 0.12 & $0.16^{*}$ & 6.79 & 0.09 & $0.13^{*}$ & 4.87 \\
\hline b* & 0.12 & $0.17^{*}$ & 2.54 & 0.10 & $0.14^{*}$ & 2.39 & 0.09 & $0.12^{*}$ & 1.83 \\
\hline
\end{tabular}

*Significant @ 5\% level 
Table.7 Influence of dyeing conditions on colour strength and colour co-ordinates of silk yarn mordanted with different mordant combinations

Dye concentration -10 per cent (owf)

\begin{tabular}{|c|c|c|c|c|c|c|c|}
\hline $\begin{array}{l}\text { Colour co- } \\
\text { ordinates }\end{array}$ & Control & $\begin{array}{l}\text { Potash } \\
\text { alum + } \\
\text { Copper } \\
\text { sulphate } \\
\text { (PA+CS) }\end{array}$ & $\begin{array}{c}\text { Potash } \\
\text { alum + } \\
\text { Stannous } \\
\text { chloride } \\
\text { (PA+SC) }\end{array}$ & $\begin{array}{l}\text { Potash alum } \\
+ \text { Copper } \\
\text { sulphate } \\
\text { (PA+FS) }\end{array}$ & $\begin{array}{c}\text { Copper } \\
\text { sulphate + } \\
\text { Stannous } \\
\text { chloride } \\
\text { (CS+SC) }\end{array}$ & $\begin{array}{l}\text { Copper sulphate + } \\
\text { Ferrous sulphate } \\
\text { (CS+FS) }\end{array}$ & $\begin{array}{c}\text { Stannous chloride }+ \\
\text { Ferrous sulphate } \\
\text { (SC+FS) }\end{array}$ \\
\hline $\mathrm{K} / \mathrm{S}$ & 15.26 & $\begin{array}{l}22.47 \\
(5.07)\end{array}$ & $\begin{array}{l}62.44 \\
(4.92)\end{array}$ & $\begin{array}{c}70.03 \\
(35.26)\end{array}$ & $\begin{array}{l}30.14 \\
(4.92)\end{array}$ & $\begin{array}{l}102.03 \\
(42.03)\end{array}$ & $\begin{array}{c}86.91 \\
(41.15)\end{array}$ \\
\hline $\mathbf{L}^{*}$ & 67.47 & $\begin{array}{c}63.17 \\
(-3.06)\end{array}$ & $\begin{array}{l}67.46 \\
(4.09)\end{array}$ & $\begin{array}{c}38.45 \\
(-28.50)\end{array}$ & $\begin{array}{l}59.29 \\
(4.09)\end{array}$ & $\begin{array}{c}32.64 \\
(-34.76)\end{array}$ & $\begin{array}{c}34.74 \\
(-32.95)\end{array}$ \\
\hline $\mathbf{a}^{*}$ & 5.64 & $\begin{array}{c}3.86 \\
(-1.58)\end{array}$ & $\begin{array}{c}5.01 \\
(-0.73)\end{array}$ & $\begin{array}{c}1.96 \\
(-3.83)\end{array}$ & $\begin{array}{c}5.81 \\
(-0.73)\end{array}$ & $\begin{array}{c}2.49 \\
(-3.09)\end{array}$ & $\begin{array}{c}2.26 \\
(-3.34)\end{array}$ \\
\hline $\mathbf{b}^{*}$ & 22.76 & $\begin{array}{l}22.40 \\
(0.43)\end{array}$ & $\begin{array}{l}24.85 \\
(2.13)\end{array}$ & $\begin{array}{c}21.59 \\
(-20.38)\end{array}$ & $\begin{array}{l}26.44 \\
(2.13)\end{array}$ & $\begin{array}{c}20.04 \\
(-22.88)\end{array}$ & $\begin{array}{c}21.85 \\
(-24.25)\end{array}$ \\
\hline
\end{tabular}

Figures in parenthesis indicate $\mathrm{K} / \mathrm{S}(\Delta \mathrm{E}), \mathrm{L}^{*}(\Delta \mathrm{L}), \mathrm{a}^{*}(\Delta \mathrm{a}), \mathrm{b} *(\Delta \mathrm{b})$

K/S- Colour strength

L-The lightness/darkness co-ordinate

$a^{*}$ - The red/green co-ordinate with $+a^{*}$ indicating red $-a^{*}$ indicating green

$b^{*}$ - The yellow/blue co-ordinate with $+b^{*}$ indicating yellow and $-b^{*}$ indicating blue

\section{ANOVA Table}

\begin{tabular}{c}
$\begin{array}{c}\text { Colour } \\
\text { co- } \\
\text { ordinates }\end{array}$ \\
\hline $\mathrm{K} / \mathrm{S}$ \\
\hline $\mathrm{L}^{*}$ \\
\hline $\mathbf{a}^{*}$ \\
\hline $\mathbf{b}^{*}$
\end{tabular}

$\mathrm{PA}+\mathrm{CS}$

$\mathrm{PA}+\mathrm{SC}$

$\mathrm{PA}+\mathrm{FS}$

$\mathrm{CS}+\mathrm{SC}$

$\mathrm{CS}+\mathrm{FS}$

\begin{tabular}{|c|c|c|c|c|c|c|c|c|c|c|c|c|c|c|c|c|c|}
\hline S.Em. \pm & $\begin{array}{c}\text { CD }(5 \\
\%)\end{array}$ & CV \% & S.Em. \pm & $\begin{array}{c}\text { CD (5 } \\
\%)\end{array}$ & CV \% & S.Em. \pm & $\begin{array}{c}\text { CD (5 } \\
\%)\end{array}$ & CV \% & S.Em. \pm & $\begin{array}{c}\text { CD (5 } \\
\%)\end{array}$ & CV \% & S.Em. \pm & $\begin{array}{c}\text { CD (5 } \\
\%)\end{array}$ & CV \% & S.Em. \pm & $\begin{array}{c}\text { CD }(5 \\
\%)\end{array}$ & CV \% \\
\hline 1.06 & $1.55^{*}$ & 12.62 & 0.63 & $0.93 *$ & 10.23 & 3.41 & $4.97 *$ & 17.87 & 3.41 & $4.97 *$ & 17.87 & 3.68 & $5.36^{*}$ & 14.02 & 2.21 & $3.23 *$ & 9.68 \\
\hline 0.89 & $1.29 *$ & 3.03 & 0.72 & $1.05^{*}$ & 2.33 & 0.79 & $1.16^{*}$ & 3.35 & 0.79 & $1.16^{*}$ & 3.35 & 0.53 & $0.78^{*}$ & 2.39 & 0.43 & $0.63^{*}$ & 1.88 \\
\hline 0.47 & $0.69^{*}$ & 4.67 & 0.28 & $0.41 *$ & 2.64 & 0.43 & $0.63 *$ & 7.89 & 0.43 & $0.63^{*}$ & 7.89 & 0.35 & $0.51 *$ & 6.84 & 0.28 & $0.40 *$ & 5.92 \\
\hline
\end{tabular}

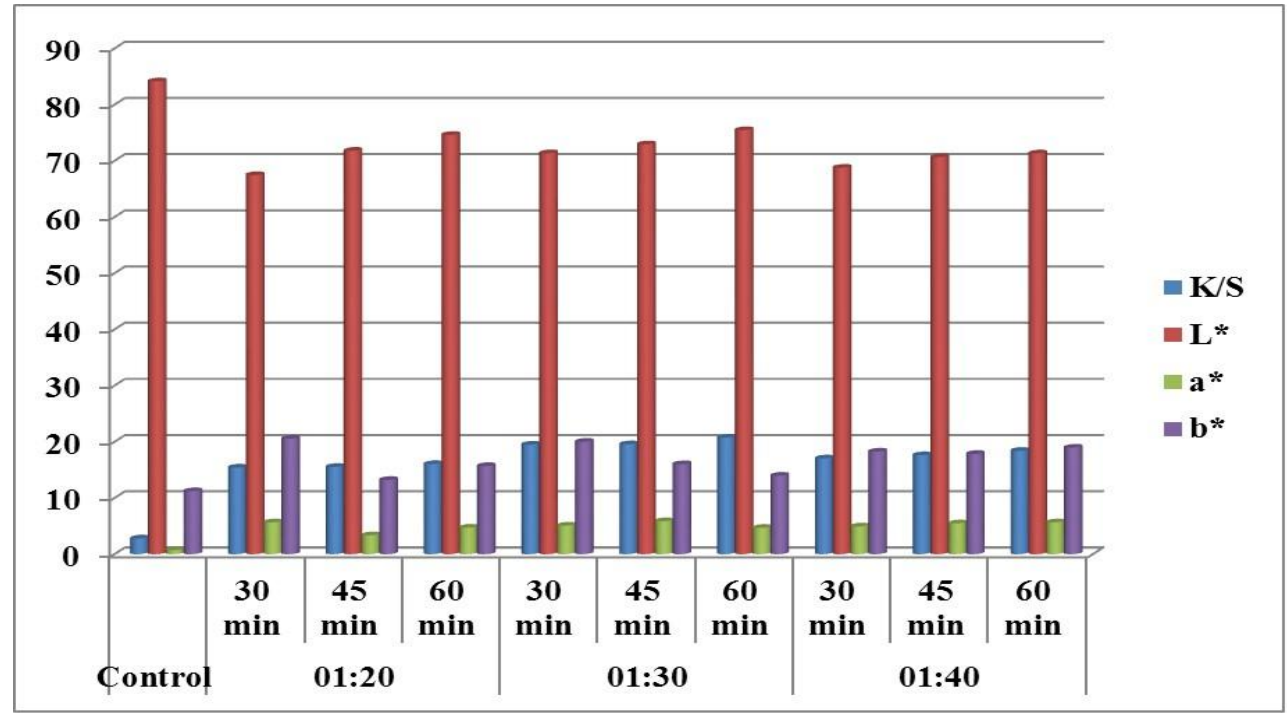

Fig 1. . Influence of dyeing conditions on colour strength and colour co-ordinates of silk yarn dyed with Acacia Nilotica pods 


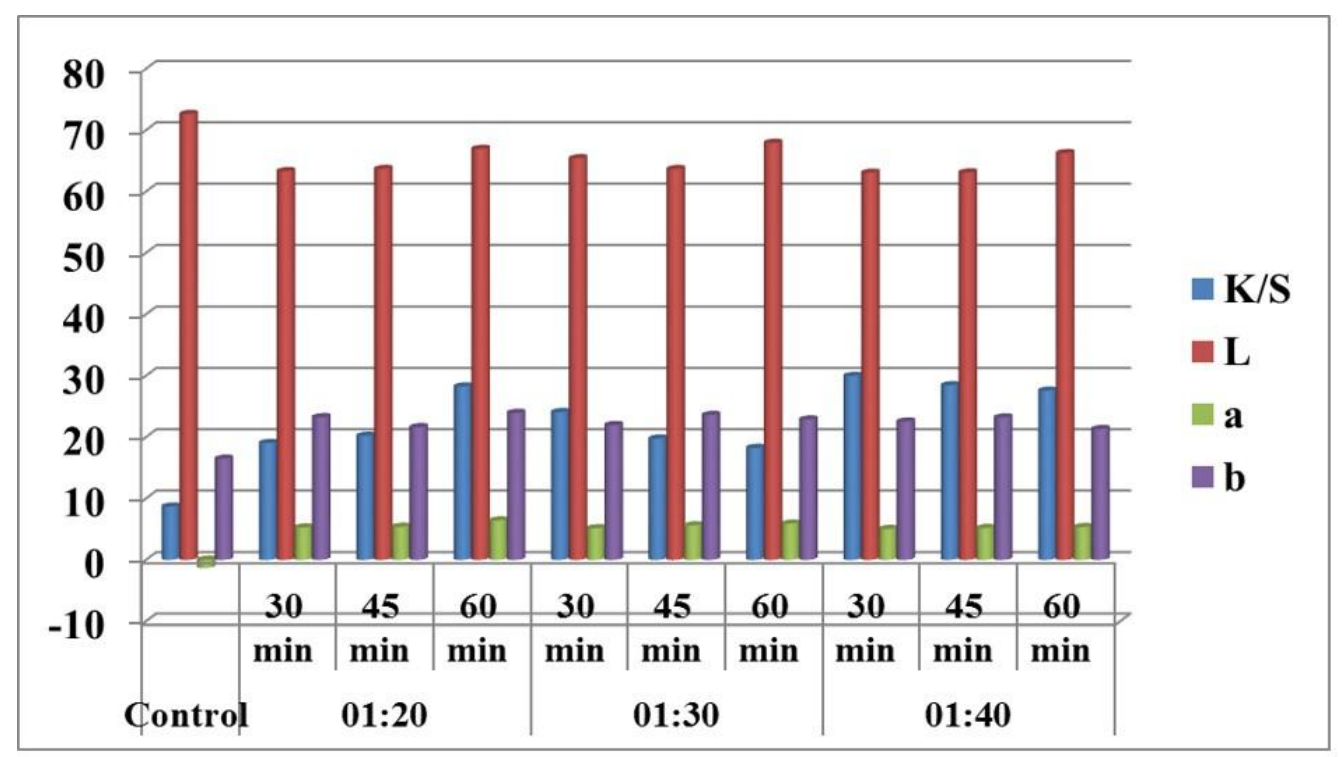

Fig 2. Influence of dyeing conditions on colour strength and colour co-ordinates of silk yarn pretreated with myrobolan

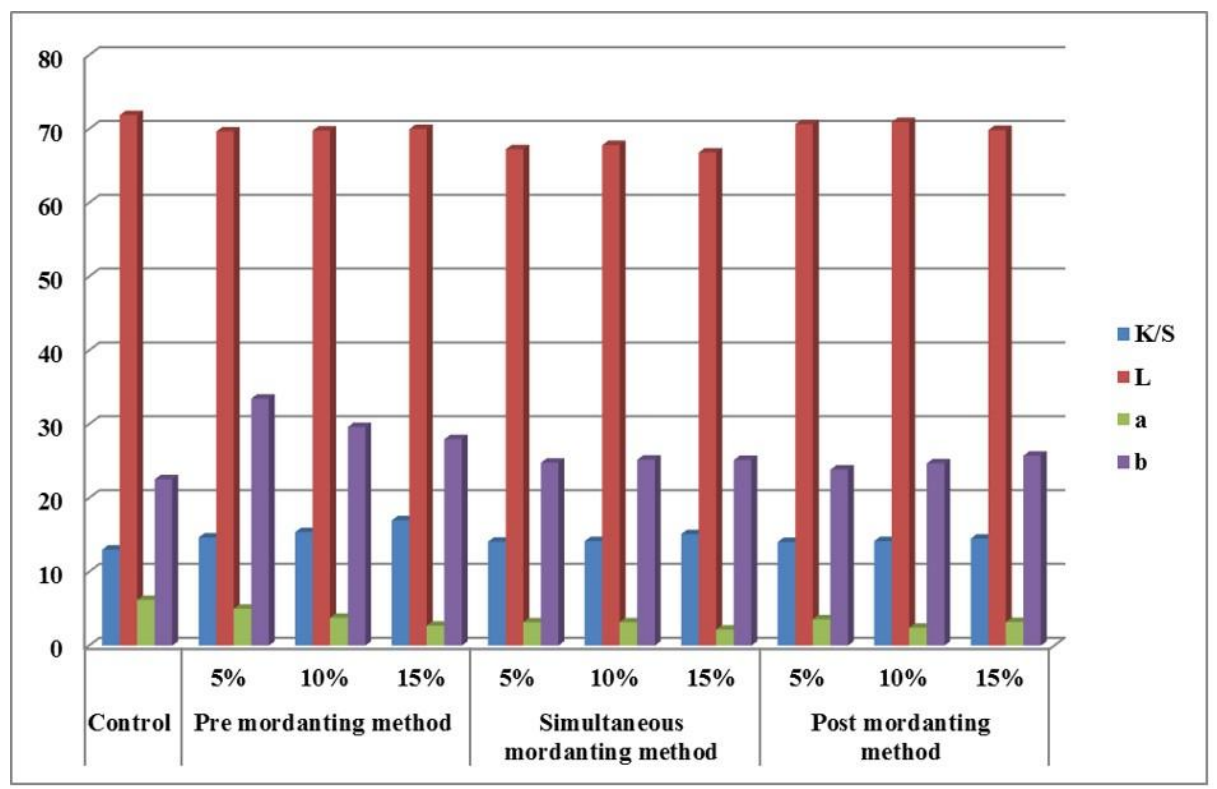

Fig 3. Influence of dyeing conditions on colour strength and colour co-ordinates of silk yarn mordanted with potash alum 


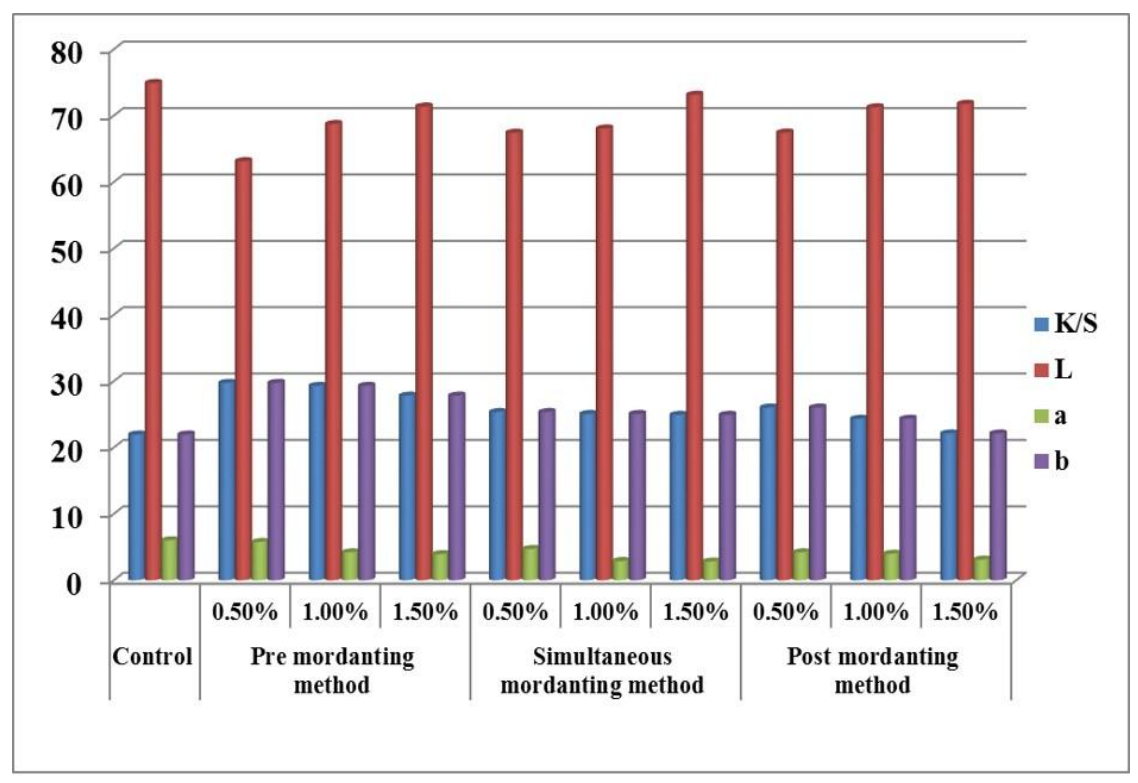

Fig 4. Influence of dyeing conditions on colour strength and colour co-ordinates of silk yarn mordanted with stannous chloride

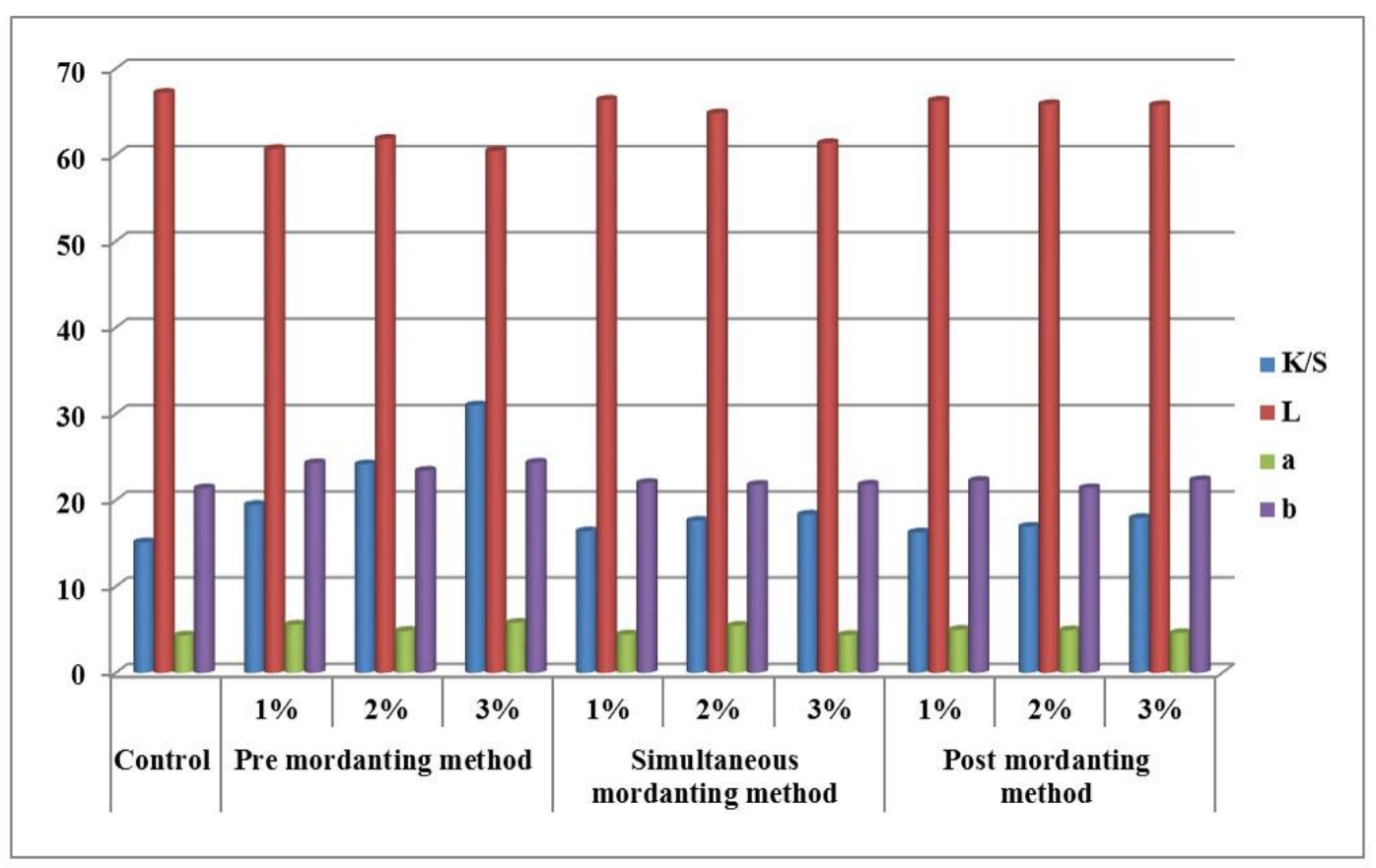

Fig 5. Influence of dyeing conditions on colour strength and colour co-ordinates silk yarn mordanted with copper sulphate 


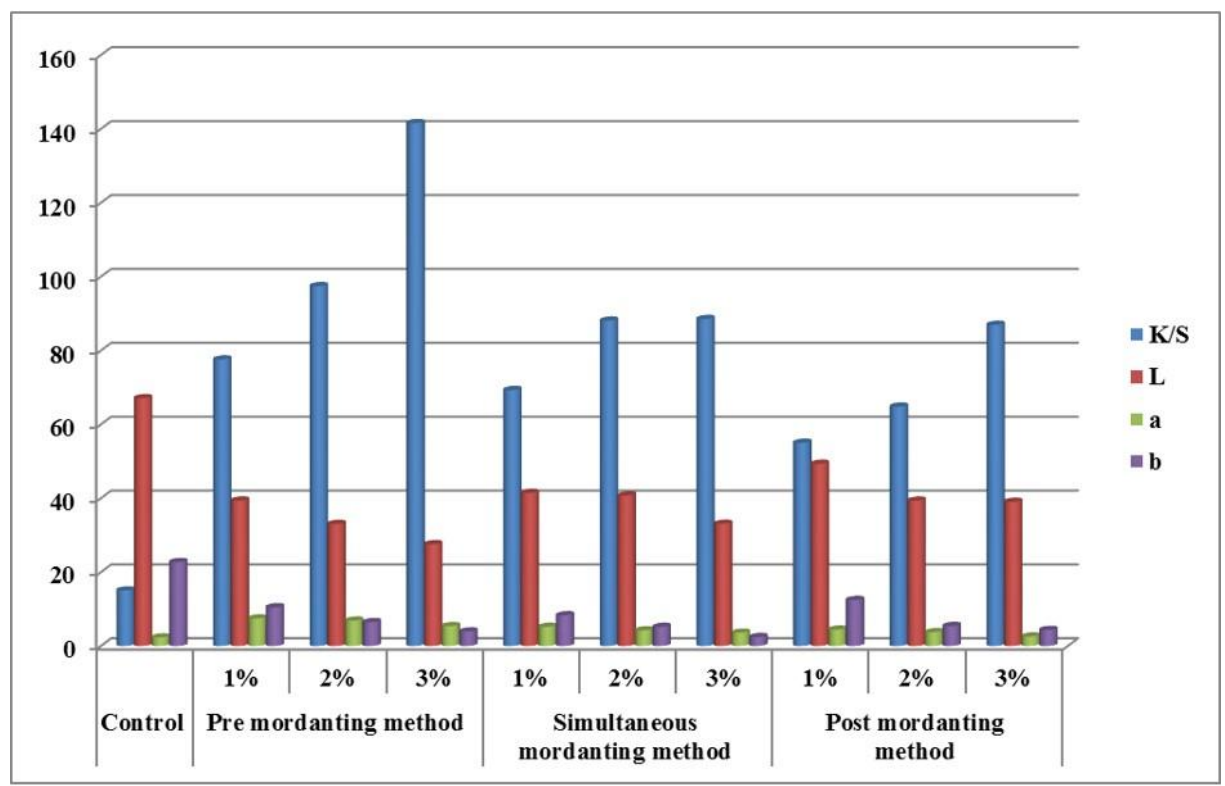

Fig 6. Influence of dyeing conditions on colour strength and colour co-ordinates of silk yarn mordanted with ferrous sulphate

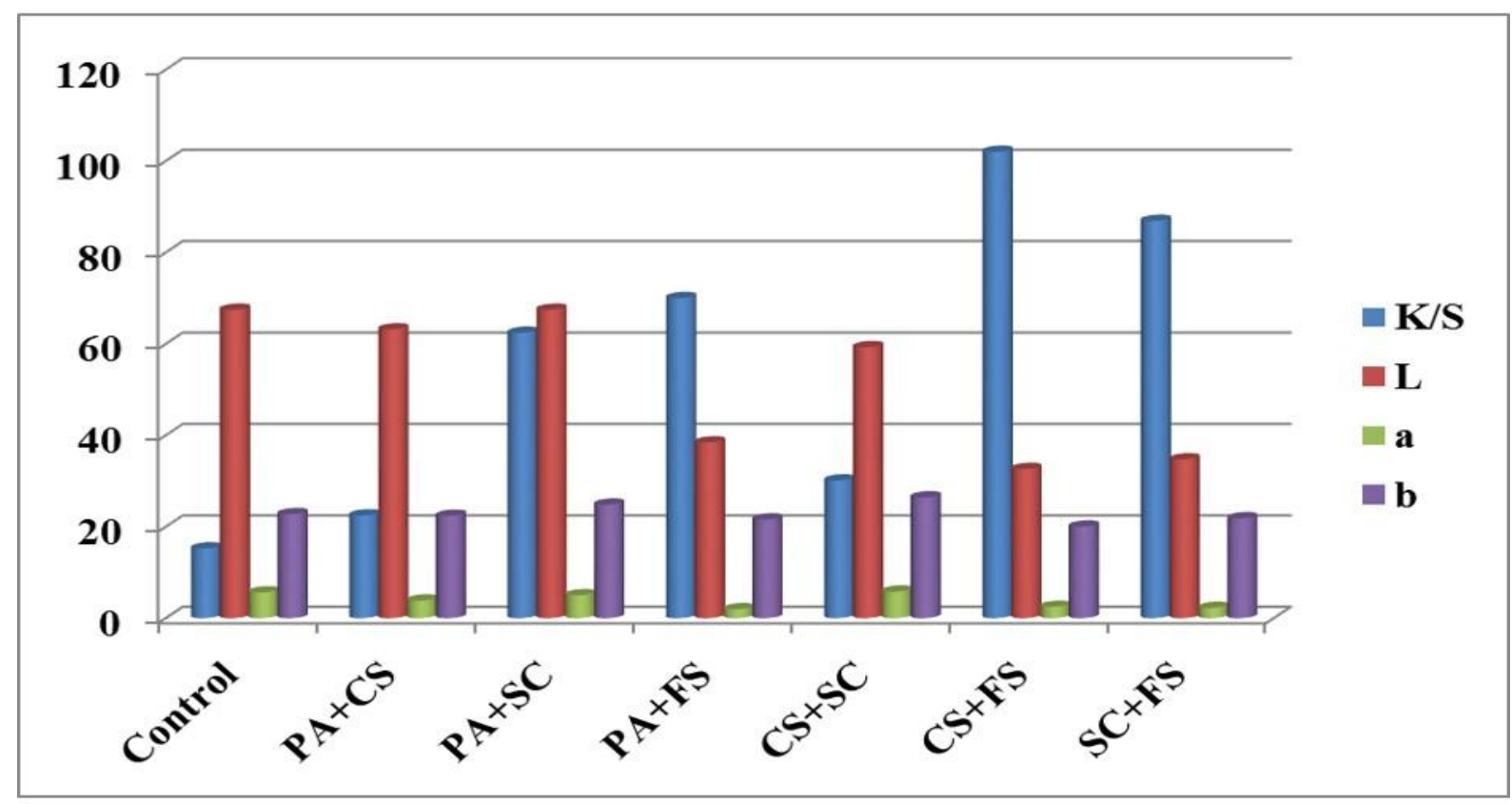

Fig 7. Influence of dyeing conditions on colour strength and colour co-ordinates of silk yarn mordanted with different mordant combinations 
This could be due to, transition metal ions of ferrous sulphate that bond strongly with natural dye molecules due to their ability to form coordination complexes and thus yields deeper shades of dyed silk yarn stated by Mongkholrattanasit (2013).

Influence of dyeing conditions on colour strength and colour co-ordinates of silk yarn mordanted with different mordant combinations

The Table 7 (Fig. 7) discloses the control sample possessed significantly least colour strength (15.26) and greater lightness value (67.47) than the silk dyed with different mordant combinations. This may be due to, mordant and mordant combinations are known to promote the binding of the dyes to fibre by forming a chemical bridge between dye and fibre, improving the staining ability of the dye along with increasing fastness property (Subhashini, 2009).

Among the mordant combinations silk yarn pre-treated with ferrous sulphate and their combinations $(\mathrm{FS}+\mathrm{CS}, \quad \mathrm{FS}+\mathrm{SC}, \quad \mathrm{FS}+\mathrm{PA})$ yields greater colour strength and darker shades than the other mordant combinations. The reason could be that, ferrous sulphate is known as green vitriol and is used for darkening /browning and blackening of the colours/ shades and is extensively used to get grey to black shades.

The colour co-ordinates of $\mathrm{a}^{*}$ and $\mathrm{b}^{*}$ values of copper sulphate + stannous chloride showed more redder (5.81) and yellower (26.44) than the control and other different mordant combinations. This may be due to, chemical composition of copper which enhances the pre-mordanted dyed silk towards redder and yellower. Copper sulphate and stannous chloride are good additives, saddens colours and brings out bright colours than the other mordants.
However, the varied M.L.R, dye concentrations and dyeing time is influences on colour strength and colour co-ordinates. Hence, the null hypothesis is accepted.

The lower reflectance and higher colour strength value was found in lower dye concentration i.e., $5 \mathrm{~g}$ and $10 \mathrm{~g}$ dye concentrations with varied M.L.R (1:20, 1:30 and 1:40) and time intervals (30, 45 and 60 $\min )$ than the 15 and $20 \mathrm{~g}$ dye concentration.

The silk yarn dyed in 1:30 dye bath at varied dyeing intervals obtained maximum colour yield i.e., $20.73(60 \mathrm{~min})$ than the 1:40 and 1:20 M.L.R in different dyeing intervals.

Myrobolan treated silk yarn dyed in 1:40 dye bath at varied dyeing intervals obtained maximum colour yield i.e., 29.95 (30 $\mathrm{min})$ than the 1:20 and 1:30 MLR in different dyeing intervals.

The silk yarn mordanted with potash alum, stannous chloride, copper sulphate and ferrous sulphate in pre-mordanting method in varied concentration showed increase in colour strength (K/S) values and obtained more redder $(+a)$ and yellower $(+b)$ shades compared to simultaneous and post mordanting method.

The silk yarn mordanted with copper sulphate +ferrous sulphate showed better colour strength (102.03) and darker shades (32.64) than the other mordant combinations. The more redder (5.81) and yellower (26.44) shades were found in copper sulphate + stannous chloride.

It can be concluded that Acacia nilotica pods are good source for dyeing silk yarn with different mordants and mordant combinations which yield vivid colour shades. A fodder source is now being used as value added eco dye source for textile coloration and will open 
up for rural folk who can take up these activities as a enterprise to sustain their livelihood.

\section{Acknowledgement}

We acknowledge our sincere thanks to Directorate of Research UAS Dharwad for funding the student fellowship during student research work.

\section{References}

Abhishek, R., Haokip, V. and Chandrawanshi, S., 2015, Acacia nilotica: a multipurpose tree and source of Indian gum arabic. South Indian Journal of Biological Sciences, 1(2): 66-69.

Khan, S.A., Ahmed, A., Khan, M.I., Yusuf, M., Shahid, N., Manzoor and Mohammad, F., 2012, Antimicrobial activity of wool yarn dyed with Rheum emodi L. (Indian Rhubarb). Dyes and Pigments, 95: 206-214.

Malviya, S., Rawat, S., Kharia, A. and Verma, M., 2011, Medicinal attributes of Acacia nilotica Linn. - A comprehensive review on ethnopharmacological claims. International Journal of Pharmacy \& Life Sciences, 2(6): 830-837.
Mongkholrattanasit, R., Klaichoi, C., Rungruangkitkrai, N., Punrattanasin, N., Sriharuksa, K. and Nakpathom, M., 2013, Dyeing Studies with Eucalyptus, Quercetin, Rutin, and Tannin:A Research on Effect of Ferrous Sulfate Mordant. Journal of Textiles, 1-8.

Samanta, A. K. and Konar, A., 2010. Dyeing of textiles with natural dyes. J Inst Engg. Text Engg., 91(7): 30-56.

Singh, O.P., 2000, Natural dyes: The Pros and Cons. The Indian Tex. J., 110(4): 42-46.

Subhashini, S., Rajalaxmi, R. And Venikeertheeswari, N., 2009, A systematic and scientific approach to the extraction and dyeing with a natural dye on silk- annato seeds dye. Orient. $J$. Chem., 25(1): 77-84.

Uddin, M.G., 2014, Effects of different mordants on silk fabric dyed with onion outer skin extracts. Journal of Textiles, 97(2): 1-8.

Wanyama, P.A.G., Kiremire, B.T., Ogwok, P. and Murumu, J.S., 2010, The Effect of Different Mordants on Strength and Stability of Colour Produced from Selected Dye-Yielding Plants in Uganda. International Archive of Applied Sciences and Technology, 1(2): 81-92.

www.ibef.Org

\section{How to cite this article:}

Dilshad Jamadar, K.J. Sannapapamma and Kasturiba, B. 2018. Influence of Dyeing Conditions on Colour Strength and Colour Co-Ordinates of Silk Yarn Dyed with Acacia nilotica Pods. Int.J.Curr.Microbiol.App.Sci. 7(08): 4756-4769. doi: https://doi.org/10.20546/ijcmas.2018.708.500 